\title{
A de novo C19orf12 heterozygous mutation in a patient with MPAN
}

Edoardo Monfrini MD ${ }^{1}$, Valentina Melzi MSc${ }^{1}$, Gabriele Buongarzone MD¹, Giulia Franco MD ${ }^{1}$, Dario Ronchi $\mathrm{PhD}^{1}$, Robertino Dilena $\mathrm{MD}^{2}$, Elisa Scola $\mathrm{MD}^{3}$, Paola Vizziello $\mathrm{MD}^{4}$, Andreina Bordoni MSc${ }^{1}$, Nereo Bresolin $\mathrm{MD}^{1}$, Giacomo Pietro Comi MD ${ }^{1}$, Stefania Corti MD $\mathrm{PhD}^{1}$, Alessio Di Fonzo MD $\mathrm{PhD}^{1 *}$.

\section{Affiliations:}

${ }^{1}$ IRCCS Foundation Ca' Granda Ospedale Maggiore Policlinico, Dino Ferrari Center, Neuroscience Section, Department of Pathophysiology and Transplantation, University of Milan, Milan, Italy.

${ }^{2}$ Servizio di Epilettologia e Neurofisiopatologia Pediatrica, UO Neurofisiopatologia, Fondazione IRCCS Ca' Granda Ospedale Maggiore Policlinico, Milan, Italy.

${ }^{3}$ Neuroradiology Unit, Fondazione IRCCS Ca' Granda Ospedale Maggiore Policlinico, Milan, Italy.

${ }^{4}$ Unità Operativa Neuropsichiatria dell'Infanzia e dell'Adolescenza, Fondazione IRCCS Ca' Granda Ospedale Maggiore Policlinico Milano, Milan, Italy.

\section{Corresponding author:}

*Alessio Di Fonzo MD PhD Neuroscience Section, Department of Pathophysiology and Transplantation (DEPT), University of Milan, Neurology Unit, IRCCS Foundation Ca’ Granda Ospedale Maggiore Policlinico, Via Francesco Sforza 35, 20122 Milan Italy. Tel: +39 0255033807; Fax: +39 0255033800; Email: alessio.difonzo@policlinico.mi.it

Word count: 823.

Key words: NBIA, MPAN, C19orf12, Heterozygous mutation, De novo mutation.

Financial disclosure: The authors have no conflicts of interests to declare.

Funding sources: This work was supported by ADF’s funds, from Intesa San Paolo and Fresco Institute.

Ethics: The relevant ethical authorities approved the study and written informed consent was obtained from all involved subjects. 


\section{Main Text}

Mitochondrial Membrane Protein-Associated Neurodegeneration (MPAN or NBIA4) is caused by biallelic mutations in the C19orf12 gene. MPAN accounts for the largest proportion of NBIA after PKAN (PANK2 mutations) and PLAN (PLA2G6 mutations). It is characterized by juvenile-onset spastic paraparesis, levodopa-unresponsive parkinsonism, dystonia and neuropsychiatric symptoms. Additional features are optic atrophy, dysphagia, dysarthria, and motor axonal neuropathy. Brain MRI displays T2-weighted symmetrical hypointensities in globus pallidus (GP) and substantia nigra (SN) [1]. Missense, frameshift and nonsense mutations were found in exons 2 and 3 of C19orf12 (NM_001031726 transcript). One splice-site mutation (c.194-2A>G) was identified in intron 2 (Figure 1a). Specific variants were found to be frequent in MPAN cases of selected populations (c.204_214del - p.G69Rfs*10 in Eastern Europeans and p.T11M in Turkish) [2, 3]. Here we report a case of NBIA with a novel C19orf12 mutation with molecular evidence of de novo occurrence

The relevant ethical authorities approved the study and written informed consent was obtained from all involved subjects. The proband is the only child of non-consanguineous Italian parents. Familial history was negative for neurological disorders (Figure 1b). She was born at term by spontaneous delivery after an uneventful pregnancy. Motor development was at first normal, while a delay in language development was reported at the age of 3. Height-weight growth has been always at the lower normal limits (3-10 centiles). At the age of 5 she developed a progressive imbalanced gait associated with lower limbs rigidity and later onset of right hand dystonia forced her to use the left hand to write. She developed mild hirsutism at 9 years (pubis and limbs) and precocious puberty. Ocular involvement was present and included low vision, hypermetropia and astigmatism. She attends high school with a support teacher for learning disability (IQ 74). She came to the attention of our outpatient clinic at the age of 16 years. The neurological examination showed moderate dysarthria, cervical dystonia, dysdiadochokinesia, mild intentional and postural upper limbs tremor, lower limbs spastic hypertonia associated with movement-exacerbated dystonic postures of feet, 
patellar hyperreflexia and bilateral Babinski sign. Typical radiological findings of SN and GP hypointensity in SWI, T2* and T2-weighted MRI with a T2-hyperintense medial medullary lamina (MML) were present, with the additional finding of subthalamic nucleus involvement. No cortical or cerebellar atrophy was evident (Figure 1d). Electroencephalogram and electroretinogram were normal, while visual evoked potential displayed an increased latency of P100 wave bilaterally with reduced amplitude and EMG showed diffuse axonal motor neuropathy. Fundus examination revealed optic atrophy.

Genetic analysis revealed a novel heterozygous C19orf12 c.265_266delAT (NM_001031726) p.M89Gfs*12 (NP_001026896) mutation in the proband, but not in her parents (Figure 1b-c). False paternity was excluded using eight polymorphic short tandem repeats (STR) on chromosome X. This suggests the p.M89Gfs*12 being a de novo mutation. Sanger sequencing of all exons and intron-exons boundaries of PANK2, PLA2G6, C19orf12, FA2H, WDR45, COASY, CP, and FTL, and full-length amplification followed by sequencing of PANK2 and WDR45 transcript did not detect other pathogenic mutations or rearrangements.

The amount of C19orf12 transcripts was measured by quantitative reverse-transcription PCR in lymphocytes of patient, parents and controls. This assay failed to detect a reduced mRNA quantity in the proband cDNA, suggesting the absence of degradation through nonsense-mRNA decay. Sequence analysis of C19orf12 transcripts revealed the presence of the heterozygous c.265_266delAT mutation in the proband and ruled-out splicing aberrations on both mutated and non-mutated alleles.

In order to explore a possible mosaicism, the mutation was studied in DNA from several tissues (hair, saliva and urine). The presence of the p.M89Gfs*12 in all tissues suggests a germ-line mosaicism in a parent.

MPAN is considered an autosomal recessive disorder; however, five monoallelic C19orf12 mutations have been already described $[1,3,4]$. A second occult mutation was suspected to be 
present in these cases. Deletion screening and promoter regions sequencing in some of these cases did not identified a second mutation [1, 3, 4]. Interestingly, all these mutations localize on exon 3 (G69R, p.Q86*, p.A94Cfs*8, p.A120Gfs*32 and p.L99fs*102), including deleterious frameshift/stop mutations on the C19orf12 C-terminal domain, as in the case described in this study. Moreover, in a family with a heterozygous p.A120Gfs*32 mutation family history suggested a possible autosomal-dominant inheritance; indeed, the father of the patient, who died at age 47 following a long course of progressive dementia with parkinsonism, showed a typical MPAN neuropathology at postmortem examination [3].

Several lines of evidence support the pathogenic role of the identified variant reported here: the association with clinical-radiological features of MPAN, the de novo occurrence of the variant, the lack of additional mutations or aberrant splice sites on C19orf12 transcripts and the absence of genetic mutations in other NBIA genes. The lack of a second mutation may indicate a dominant negative effect of this variant. Alternatively, the combination of this p.M89Gfs*12 with mutations in other still unknown causative genes may be hypothesized. In this view, studies on additional MPAN cases with monoallelic mutations are required in order to explore their role in NBIA.

\section{Figure Legend}

A) Representation of the C19orf12 gene mutations reported in MPAN phenotypes so far. B) Pedigree of the family under study. Black symbol denotes affected individual. C) Electropherograms of the mutation c.265_266delAT of C19orf12 gene in the proband (II.1) and wild type sequence in her parents (I.1 and I.2). D) Patient Brain MRI (T2, FLAIR and SWI sequence), performed at the age of 16 , showing hypointensity of substantia nigra, globus pallidus and interestingly, subthalamic nucleus. (Abbreviations: wt =Wild type; mut = Mutated; GP = Globus Pallidus; GPe = External Globus Pallidus; GPi = Internal Globus Pallidus; SN = Substantia Nigra; STN = Subthalamic nucleus; MML = Medial Medullary Lamina). 


\section{REFERENCES}

1. Hartig, M., et al., Mitochondrial membrane protein-associated neurodegeneration (MPAN). Int Rev Neurobiol, 2013. 110: p. 73-84.

2. Olgiati, S., et al., The p.Thr11Met mutation in c19orf12 is frequent among adult Turkish patients with MPAN. Parkinsonism Relat Disord, 2017. 39: p. 64-70.

3. Hogarth, P., et al., New NBIA subtype: genetic, clinical, pathologic, and radiographic features of MPAN. Neurology, 2013. 80(3): p. 268-75.

4. Panteghini, C., et al., C19orf12 and FA2H mutations are rare in Italian patients with neurodegeneration with brain iron accumulation. Semin Pediatr Neurol, 2012. 19(2): p. 75-81. 


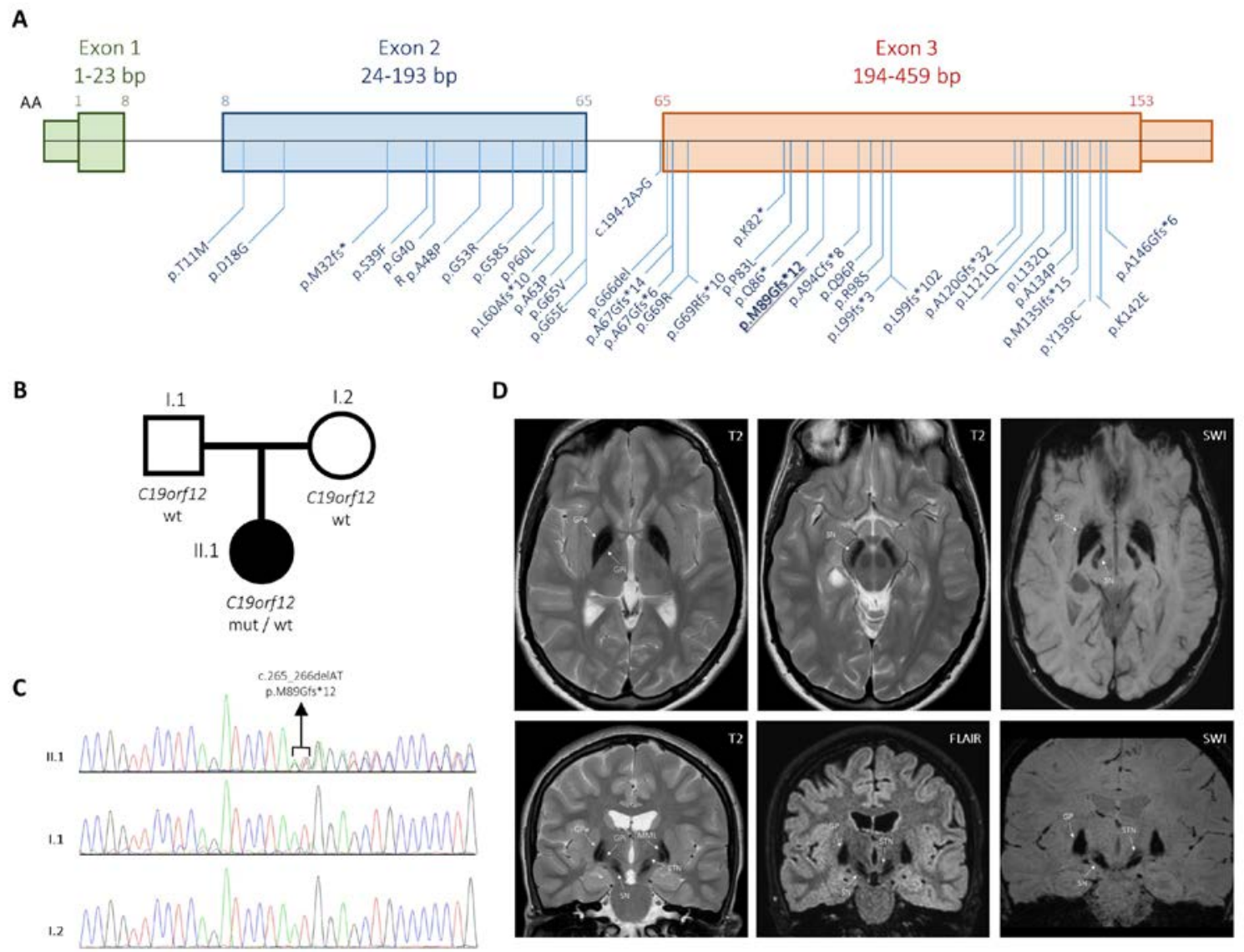

